\title{
Optimizing Sampling Schemes for Electron Tomography: Dual- and Multiple-Axis Tomography
}

Georg Haberfehlner, Raphaël Serra, David Cooper, Guillaume Audoit, Sylvain Barraud and Pierre Bleuet

\section{CEA, LETI, MINATEC Campus, 17 rue des Martyrs, 38054 GRENOBLE Cedex 9, France}

Electron tomography extends the potential of a transmission electron microscope (TEM) from imaging of single images to three-dimensional (3D) imaging by reconstructing objects from their twodimensional projections. The resolution in electron tomography is generally limited by the number of projections, which can be taken of an object. This limitation arises due to beam sensitivity of many samples, due to limited accuracy of the stage mechanics as well as due to time constraints. Approaches to improve the resolution for a given number of projections have been developed, such as discrete tomography [1] and reconstruction techniques based on minimization of the total variation of images [2, 3]. These approaches use prior knowledge about the sample as constraints to improve the reconstruction.

By contrast dual-axis tomography has been used mostly for its capability of reducing missing wedge artifacts, which appear in tomography, if the geometry of the sample or the sample holder does not allow rotation over a full tilt range of $180^{\circ}[4,5]$. In materials science, in particular for focused ion beam (FIB)-based sample preparation, sample geometries and sample holders have been developed, which can eliminate the missing wedge and might therefore give the impression of rendering dual-axis tomography useless for such applications.

Here we show that dual-axis tomography can also serve to improve the resolution of a reconstruction without or with a small missing wedge, in particular for samples which contain features with high aspect-ratio, which are small in one dimension and large in the other dimensions. We employ the dense sampling by tomography in the direction of the tilt axis, which stems from the fact, that each slice along a tilt axis can be reconstructed independently of its neighboring slices. If sample features change slowly along directions perpendicular to a tilt axis, these features can be reconstructed with the original 2D resolution of the images in the direction of a tilt axis, without the need for using any prior knowledge for the reconstruction.

Samples which fulfill these conditions are often encountered in nanotechnology, as many device concepts, also for complex 3D structures are based on deposition of thin films. For such devices it is then possible to adapt the tilt-axes accordingly to optimize the resolution perpendicular to these layers. Thickness and roughness of these layers can then be measured with high accuracy.

We used dual-axis electron tomography to get a high resolution 3D reconstruction of a tri-gate transistor [6]. The gate of a tri-gate transistor consists of three approximately perpendicular surfaces, one oriented horizontally and two vertically, which surround the channel. To optimize the resolution for all gate surfaces we oriented two tilt-axes perpendicular to the gate surfaces. The first tilt-axis is oriented perpendicular to the horizontal gate surface. Fig. 1a shows a reconstruction from the first tilt series where the horizontal gate surface is well defined, but the resolution for the two vertical surfaces is reduced. The second tilt-axis is oriented perpendicular to the vertical gate surfaces, in the corresponding reconstruction (Fig. 1b) the vertical gate surfaces are well defined. 
To combine the two tilt series an alignment process based on 3D cross-correlation was applied and reconstruction is done with a dual-axis SIRT algorithm. Fig. 1c shows the combined dual-axis reconstruction. In this reconstruction all gate surfaces are well resolved, thus proofing the capability of dual-axis tomography for this kind of applications. The dual-axis reconstruction allows segmentation of the gate stack, to separate the gate oxide ( $\mathrm{HfSiON})$ and the metal gate (TiN) from other materials. Results of the segmentation are shown in Fig. 2.

The proposed approach can be easily extended to more than two axes, which will allow improving the reconstruction of complex objects by adapting multiple tilt-axes to their geometry [7].

References:

[1] K J Batenburg et al, Ultramicroscopy 109 (2009), p. 730.

[2] Z Saghi et al, Nano Letters 11 (2011), p. 4666.

[3] B Goris et al, Nature Materials 11 (2012), p. 930.

[4] D Mastronade, Journal of Structural Biology 120 (1997), p. 343.

[5] I Arslan et al, Ultramicroscopy 106 (2006), p. 994.

[6] S Barraud et al, IEEE Electron Device Letters 33 (2012), p. 1526.

[7] This work was supported by the French "Recherche Technologie de Base" (RTB) program and partially carried out in the frame of the ST/IBM/LETI joint program. The authors acknowledge access to the nanocharacterization platform (PFNC) at Minatec Campus in Grenoble.

(a)

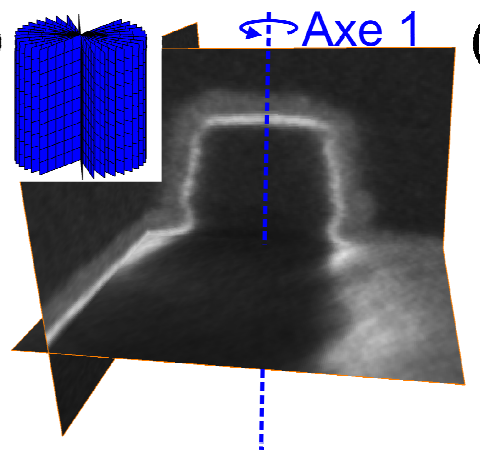

(b)

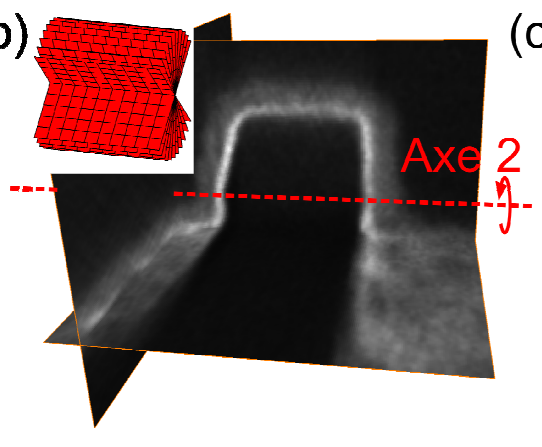

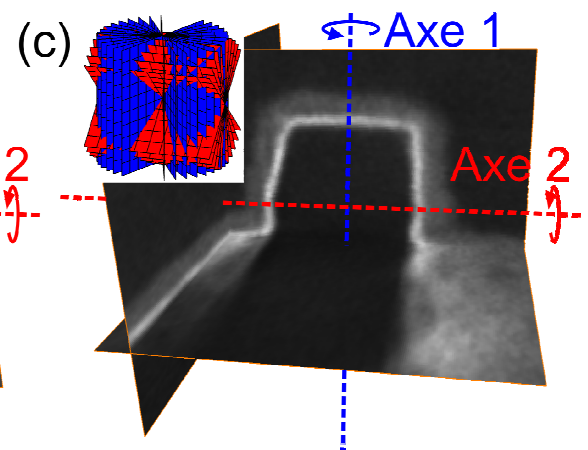

Figure 1. Orthogonal slices through single-axis $(a, b)$ and dual-axis (c) reconstructions of a tri-gate transistor. The insets in the upper left corners indicate the corresponding sampling in Fourier space.

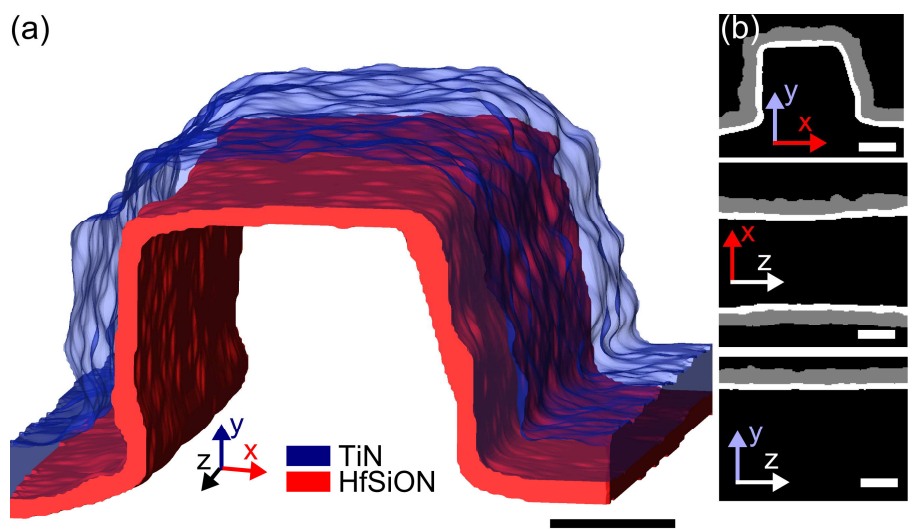

Figure 2. Segmentation of the reconstruction of a tri-gate transistor based on dual-axis reconstruction. (a) Surface rendered view, (b) Orthogonal slices through the reconstruction, scale bars are $10 \mathrm{~nm}$. 